\title{
Article \\ Performance of Transmit Aperture Selection to Mitigate Jamming
}

\author{
Isha Chauhan *,+ (D) and Manav R. Bhatnagar ${ }^{\dagger}$ \\ Department of Electrical Engineering, Indian Institute of Technology Delhi, New Delhi 110016, India; \\ manav@ee.iitd.ac.in \\ * Correspondence: Isha.Chauhan@ee.iitd.ac.in \\ + These authors contributed equally to this work.
}

Citation: Chauhan, I.; Bhatnagar,

M.R. Performance of Transmit

Aperture Selection to Mitigate Jamming. Appl. Sci. 2022, 12, 2228. https://doi.org/10.3390/ app12042228

Academic Editor: Fabrizio Granelli

Received: 28 September 2021

Accepted: 17 November 2021

Published: 21 February 2022

Publisher's Note: MDPI stays neutral with regard to jurisdictional claims in published maps and institutional affiliations.

Copyright: (c) 2022 by the authors. Licensee MDPI, Basel, Switzerland. This article is an open access article distributed under the terms and conditions of the Creative Commons Attribution (CC BY) license (https:// creativecommons.org/licenses/by/ $4.0 /)$

\begin{abstract}
In this work, we investigate the impact of the transmit aperture selection technique to alleviate the effect of jamming on a free space optical (FSO) communication system. We consider the FSO system to suffer from a jamming signal and Gaussian noise concurrently. The analysis in this paper is conducted on the assumption that all wireless optical links follow the negative exponential distribution with pointing error. A general $N \times 1$ FSO system is studied, which allows the transmitter to select a link with the maximum channel gain to transmit the information. We derive a closed-form expression of the bit error rate (BER) of the considered FSO system in the presence of a jammer. We extract the analytical coding gain and diversity order from the asymptotic behavior of the derived BER. We show that the transmit aperture selection allows the FSO system corrupted by a jammer to attain a diversity order of $0.5 \mathrm{~N}$, equal to that of a multiple-input single-output FSO system, while appreciably reducing the power requirements of $(\mathrm{N}-1)$ transmitters. The theoretical analysis is verified by simulations. We also make a quantitative comparison with repetitive coding (RC) and optical space shift keying (OSSK) and show that the transmit aperture selection has a $3 \mathrm{~dB}$ advantage over RC; moreover, for a $2 \times 1$ system, it provides twice the diversity offered by OSSK. The tenacity of aperture selection is also studied numerically for Gamma-Gamma fading FSO channels.
\end{abstract}

Keywords: aperture selection; bit error rate (BER); jamming; multiple-input single-output (MISO); negative-exponential fading; pointing error $(\mathrm{PE})$

\section{Introduction}

The past few years have witnessed enormous attention from academia and industry to free-space optical (FSO) communication. This is primarily due to advantages, such as unlicensed spectrum, higher speed, low implementation cost, and many more [1]. These benefits have led to the extensive application of FSO technology, from terrestrial to satellite communication. However, there exist a few challenges in FSO communication such as atmospheric turbulence (AT) and pointing error (PE) [2]. AT occurs due to a random change in the refractive index of air with time, classified into weak, moderate, strong, and saturation. On the other hand, PE is an aftereffect of the misalignment of the transmitter and receiver, leading to the loss of line-of-sight between the two. It causes error enhancement or complete failure of an FSO link, depending upon its severity [3]. Many techniques have been devised to improve the FSO communication.

Multiple-input multiple-output (MIMO) communication techniques are proposed to scale down the effect of channel fading due to AT and PE in the optical channel [4-7]. It is a requisite to improve the diversity order (equitable to the number of signals at the receiver enduring deep fade independently) while also pruning the system complexity and power requirements. Transmit aperture (LEDs/LASERs) selection is an attractive low-cost and low-complexity spatial diversity technique that can significantly increase the quality of the received signal and enhance the immunity to turbulence-induced fading in FSO systems [8,9]. In this technique, a single transmit aperture, which experiences 
the maximum channel gain, is selected for uncoded transmission during a transmission interval; all other apertures are kept inactive [10]. The antenna selection technique is also favored for FSO systems, as it outperforms the repetition coding (RC) scheme [11].

However, the inherent property of FSO communication being unregulated bears consequences in the form of it being vulnerable to any unsolicited network that may disrupt or attack the legitimate flow of information [12]. An FSO communication system's susceptibility to jammers mainly comes from two reasons: a confined range of operating wavelength and broad field-of-view (FoV). Typically, an FSO system is allowed to use limited wavelengths - usually, either $1300 \mathrm{~nm}$ or $1550 \mathrm{~nm}$ [13] to tackle attenuation and satisfy the eye-safety rules-making it not very laborious to access its operating wavelength. Moreover, FoV is the solid angle through which a detector is exposed to an incoming optical signal [1]. A receiving aperture's FoV is often kept broad as it tackles the fading in the channel due to AT and PE. Many terrestrial applications such as storage area networks, enterprise connectivity, etc., require aperture averaging and a wide FoV, despite the transceiver's fixed position to mitigate the effects of building sway, seismic activity, or physical obstruction [14]. In satellite communication, wider FoV is even more essential since the transceivers are mobile and very far from each other [15-18].

Having said that, a wider FoV gives room to any jammer to compromise an FSO link's security or cause complete link failure. Furthermore, as FSO transceivers are placed many feet above the ground, even for terrestrial communication, it is easy to locate them and establish a jammer in their FoV. The jammer can be a malignant optical transmitter with very large power at its disposal to increase the bit error of the authentic communication of user information among FSO transceivers. It can transmit a pulse towards the receiver to disrupt the communication as it is a challenge to decipher the legitimate signal from the jammed received signal. Hence, it is imperative to study jamming in an FSO communication system and devise different techniques to combat it.

In the literature, the general mechanism of FSO communication has been studied by [19]. In [20], a proficient discussion on FSO communication is presented. Ref. [21] focused on an FSO system in a maritime environment and conducted experimental analysis of its performance. The AT is tackled using adaptive optics in [22].

In $[23,24]$, a selection combining technique has been used to improve the quality of FSO links. A switching scheme to toggle between radio frequency (RF) and the FSO system has been proposed for hybrid a RF-FSO system in [25]. However, the effect of PE and jammers has not been studied in these works. In [26], a multiple-input single-output (MISO) FSO system is studied in the presence of an eavesdropper, and the secrecy rate is calculated. However, AT is not taken into consideration here. In [27], an FSO system with a jammer was studied for optical space shift keying (OSSK). Ref. [28] presented the application of the fault location method based on a deep neural evolution network to improve the accuracy of fault location in cloud data center interconnection. While it is a novel idea, it increases the hardware challenge in complexity and cost manifold. This work favors the transmit aperture selection technique due to its low implementation complexity and prowess in evaluating errors while simultaneously reducing power requirements. In [29], the authors addressed the issue of signal crosstalk and fragmentation of spectrum during resource allocation. Since the transmit aperture selection technique employs only one transmit aperture to disseminate the user information, the problems of crosstalk and spectral fragmentation diminish inordinately in contrast to a MISO system. Nonetheless, the transmit aperture selection technique has not been studied so far for an FSO system under the attack of a jammer.

The discussion above motivates us to study an $N \times 1$ MISO-FSO system in outdoor atmospheric surroundings, under the jammer's attack, by analyzing its bit error rate (BER) performance and studying its asymptotic behavior. The novelty of this work is as follows:

- The jamming signal is not considered to be Gaussian noise; instead, an inclusive study is carried out where additive Gaussian noise and noise from a jamming signal due to random AT are considered. 
- We consider a random jammer that becomes active with a probability of $\rho$ during a particular instance of time and stays silent for the rest of the time frame with probability $1-\rho$. Thus, the study includes partial-band and broadband jamming.

- We derive an analytical expression of probability distribution function (PDF) of a maximum of $N$ channel fading coefficients that follow the negative exponential model for saturated AT with PE.

- We calculate the PDF of the received signal at the receiver of an MISO-FSO system employing transmit aperture selection. Based on the derived expression, a closed-form expression of BER is calculated.

- A comparative study is made for different degrees of severity of PE and activity of the random jammer.

- The coding gain and diversity order are calculated to examine the system's asymptotic behavior under the influence of both noises.

- We also examine the considered system over the Gamma-Gamma (GG) fading to study the efficacy of aperture selection to combat jammers when AT conditions vary.

The rest of the paper is organized as follows. Section 2 introduces the system model and builds up the mathematical framework of the work. In Section 3, error analysis is carried for a generalized $N$ user transmitters with a jammer, and asymptotic behavior of the same is studied. The numerical results and discussion are presented in Section 4. Section 5 concludes the work.

\section{Preliminaries}

As shown in Figure 1, we consider an FSO system with $N$ transmitting apertures (LEDs/LASERs) that transmit an on-off keying (OOK) modulated optical signal, $x$, an optical receiver, and a jammer that transmits a variable energy jamming signal to disrupt the legitimate communication. The channel is modeled for saturated AT with PE, which deteriorates the authorized and jamming signal likewise. During a transmission interval, the receiver selects the signal from the transmitting aperture having the most robust signal strength.

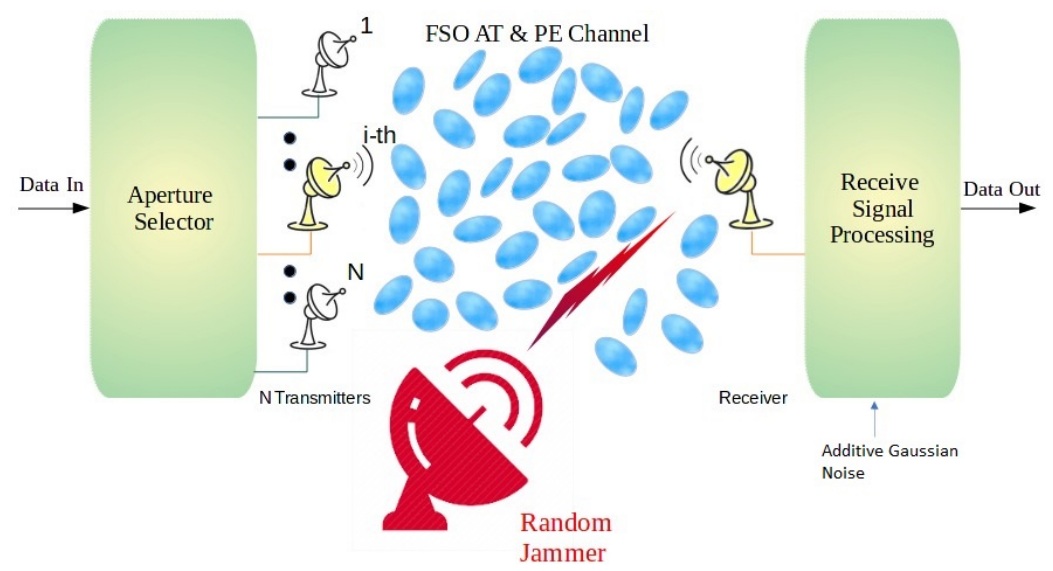

Figure 1. System model.

At the receiver, a photodiode with responsivity $R$ is used; we take the value of $R$ as unity throughout our calculations. The input-output relation can be mathematically written as

$$
y=R \sqrt{E_{x}} h_{m} x+R \sqrt{N_{j}} h_{j} J+\eta .
$$

Here, $E_{x}$ represents the peak electrical transmitted power of the legitimate transmitter. This study has considered a random jammer that alters its radiating power randomly to intercept the licensed transmission. Hence, the net contributing jamming power is $N_{j}=\frac{E_{j}}{\rho}$, where $E_{j}$ is the average jamming power. The degree of activity of the jammer is caught 
by $\rho \in[0,1]$. The random jammer has two states: active and idle state. It toggles between them with probability $\rho$, following Bernoulli's distribution. Thus, we can write

$$
P(J=1)=\rho ; \quad P(J=0)=1-\rho,
$$

where $P(\cdot)$ is the probability function, and $J$ is the jammer's state. In this paper, the random jammer is modeled to impact the authentic signal promptly and randomly. Moreover, $h_{m}$ represents the channel fading of a path between the transmitter and receiver that optimizes the system's performance. In other words, we can say

$$
h_{m}=\max \left\{h_{u_{i}}\right\}
$$

where $h_{u_{i}} \in \Re, i \in 1,2 \ldots N$ is the channel fading coefficient between the $i$ th transmitter and receiver. Furthermore, $h_{j}$ denotes the channel fading between the jammer and receiver; it is assumed that $h_{u_{i}} \mathrm{~s}$ are independent and identically distributed (i.i.d.) random variables (RVs). In addition, the legitimate and jamming channels are assumed to be 'quasi-static'. $\eta \in \Re$ represents the additive Gaussian noise with zero mean and $N_{o}$ variance. It is noted that a jammer takes no account of eye safety rules obligatory in FSO transmission and increases its transmitting power perilously to obstruct the communication of user information. Therefore, the jamming noise takes precedence over the Gaussian noise. Mathematically, we can infer the average signal-to-jamming-plus-noise ratio (SJNR) without conditioning over the fading channels, denoted by $\gamma$, as

$$
\gamma=\frac{E_{x}}{E_{j}+N_{0}}
$$

Let $E_{x}$ be taken to be of unit power. Hence, when the jammer is active $(J=1)$, we have $E_{j} \gg N_{0}$; consequently, SJNR reduces to the signal-to-jamming ratio (SJR) as $\gamma=\frac{1}{E_{j}}$. Conversely, for an idle jammer $(J=0)$, we know the power level alters to $N_{0} \gg E_{j}$; thus, we obtain a signal-to-noise ratio (SNR) as $\gamma=\frac{1}{N_{0}}$. To expedite the mathematical computation, it is assumed that $N_{0}=\frac{E_{j}}{\kappa}$, where $\kappa$ is an arbitrary positive real number. Therefore, we can construe that the legitimate information carrying signal is distorted by the jamming signal whenever the jammer is in active state. For an idle jammer, the signal only suffers from the Gaussian noise.

\subsection{Optical Channel Model}

In this work, the combined effect of AT and PE is studied for performance evaluation. The PDF of an FSO channel is modeled as

$$
h_{s}=h_{a, s} h_{p, s}
$$

where $h_{s}, s \in\left\{u_{i}, j\right\}$ defines the channel fading coefficients for an authentic user and jammer, $h_{a, s}$ is the attenuation due to AT, and $h_{p, s}$ is the attenuation due to PE. The jamming channel is considered to be optical as well, since we have considered the jammer to be an optical transmitter which aims to disrupt an authorized FSO communication. The saturated $\mathrm{AT}$ is defined by negative exponential statistics, i.e., the PDF of $h_{a, s}$ is [2]

$$
f_{h_{a, s}}\left(h_{a, s}\right)=\frac{1}{\lambda} \exp \left(-\frac{h_{a, s}}{\lambda}\right) .
$$

Here, $\lambda=\mathbb{E}\left[h_{a, s}\right]=1$, and $\mathbb{E}[\cdot]$ is the expectation operator. The PDF of $h_{p, s}$ is given by [30]

$$
f_{h_{p, s}}\left(h_{p, s}\right)=\frac{\zeta_{s}^{2}}{A_{0}^{\zeta_{s}^{2}}} h_{p, s}^{\zeta_{s}^{2}-1}, \quad 0 \leq h_{p, s} \leq A_{0}
$$


where $A_{0}=[\operatorname{erf}(v)]^{2}, v=\sqrt{\pi / 2} a / w_{z}, \zeta_{s}=w_{z e q} / 2 \sigma_{s}, a$ represents the radius of the detection aperture, $w_{z}$ denotes the beam-waist (radius calculated at $\left.e^{-2}\right), w_{z e q}^{2}=w_{z}^{2} \sqrt{\pi} \operatorname{erf}(v)$ $/\left[2 v \exp \left(-v^{2}\right)\right]$ is the equivalent beam-width at the receiver, $\sigma_{s}$ is the pointing error displacement standard deviation (jitter) at the receiver, and $\operatorname{erf}(\cdot)$ is the error function [31]. Since $\zeta_{s}$, which characterizes the PE, is inversely proportional to $\sigma_{s}$, the smaller value of $\zeta_{s}$ would symbolize greater PE severity. Now, the combined PDF of $h_{s}$ can be calculated by

$$
f_{h_{s}}\left(h_{s}\right)=\int f_{h_{s} \mid h_{a, s}}\left(h_{s} \mid h_{a, s}\right) f_{h_{a, s}}\left(h_{a, s}\right) d h_{a, s}=\frac{\zeta_{s}^{2}}{A_{0}^{\zeta_{s}^{2}}} h_{s}^{\zeta_{s}^{2}-1} \Gamma\left(1-\zeta_{s}^{2}, \frac{h_{s}}{A_{0}}\right)
$$

where $\Gamma(\cdot)$ is the incomplete gamma function [32]. In the considered FSO system, the jammer needs to transmit an optical signal to interrupt the regular communication; hence, the jamming channel is also regarded as optical.

\subsection{Bit Error Rate}

The error performance of the defined FSO system is dependent on two different potential events: the first is that the jammer is active $(J=1)$, making the jamming signal the foremost noise, and the second event is the idle jammer $(J=0)$, resulting in Gaussian noise being prominent. As is shown in Figure 2, we can calculate the bit error events for jamming and Gaussian noise through their respective distribution. The probability of occurrence of two events is $\rho$ and $(1-\rho)$, respectively, which is the same as the activity of the jammer. Now, for the event of the jammer being ON, the probability of error can be obtained by using the PDF of the received signal $y$ when $x$ is transmitted in the jamming environment. The generalized expression for $B E R$ for an active jammer can be mathematically written as

$$
B E R_{\mathrm{J}_{\mathrm{active}}}=P(x=1) P(e \mid x=1)+P(x=0) P(e \mid x=0),
$$

where $e$ implies bit error events. Taking up the case of the jammer being in the OFF state, the BER contributed by Gaussian noise for OOK signaling is [33]

$$
B E R_{\mathrm{J}_{\mathrm{idle}}}=Q\left(\frac{h_{m} \sqrt{\kappa \gamma}}{2}\right)
$$

where $Q(\cdot)$ is the $Q$-function [34]. Using (9) and (10) along with the respective probability of the jammer's activity, we attain the overall $B E R$ for the given system:

$$
\begin{aligned}
B E R= & P(J=1)[P(x=1) P(e \mid x=1)+P(x=0) P(e \mid x=0)] \\
& +P(J=0) Q\left(\frac{h_{m} \sqrt{\kappa \gamma}}{2}\right) .
\end{aligned}
$$

The (11) is a generalized expression for the BER of the given FSO system employing aperture selection.

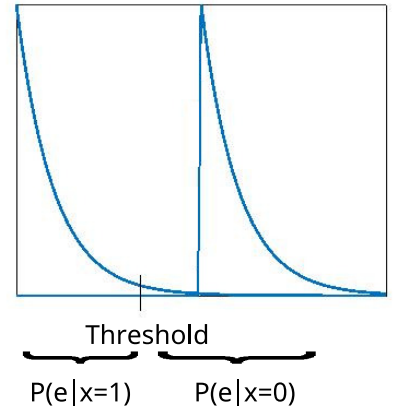

(a)

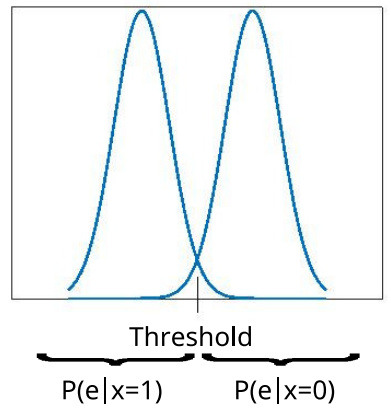

(b)

Figure 2. Probability of error events for (a) jamming noise and (b) Gaussian noise. 


\subsection{Coding Gain and Diversity}

The coding gain signifies the relative horizontal shift in the BER versus the SJNR curve at asymptotic values of SJNR, whereas the diversity order is defined as the slope of fall of the same curve at high values of SJNR. Mathematically, we can write the asymptotic BER expression as [27]

$$
\lim _{\gamma \rightarrow \infty} \operatorname{BER}(\gamma) \propto\left(G_{c} \gamma\right)^{-G_{d}},
$$

where $G_{c}$ corresponds to the coding gain, while $G_{d}$ symbolizes the diversity order of the defined FSO system.

\section{ABER Performance of Transmit Aperture Selection under Jamming}

In this section, a closed-form expression of average BER (ABER) is determined. Let us define an RV as

$$
w \triangleq \sqrt{\frac{E_{j}}{\rho}} h_{j}=\sqrt{N_{j}} h_{j},
$$

that encapsulates the jamming component at the receiver while the jammer is $\mathrm{ON} ; h_{j}$ follows the PDF defined in (8). We can calculate the PDF of RV $w$ by employing the transformation of RV [35]; thus, we can write

$$
f_{W}(w)=\frac{\zeta_{j}^{2}}{\sqrt{N_{j}} A_{0}^{\zeta_{j}^{2}}}\left(\frac{w}{\sqrt{N_{j}}}\right)^{\zeta_{j}^{2}-1} \Gamma\left(1-\zeta_{j}^{2}, \frac{w}{A_{0} \sqrt{N_{j}}}\right) U(w) .
$$

Here, $\zeta_{j}$ indicates the PE faced by the jammer at the receiver, and $U(\cdot)$ is the unit step function. Now, as we know that Gaussian noise is negligible compared to the jamming noise for $(J=1)$, we can re-write the jamming noise component at the receiver in (1) as $w=y-\sqrt{E_{x}} h_{m} x$. Substituting $w$ in (14), we obtain the PDF of the received signal $y$ for $(J=1)$ as

$$
\begin{aligned}
f_{Y}\left(y \mid \sqrt{E_{x}}, h_{m}, x\right)= & \frac{\zeta_{j}^{2}}{\sqrt{N_{j}} A_{0}^{\zeta_{j}^{2}}}\left(\frac{y-\sqrt{E_{x}} h_{m} x}{\sqrt{N_{j}}}\right)^{\zeta_{j}^{2}-1} \Gamma\left(1-\zeta_{j}^{2}, \frac{\left(y-\sqrt{E_{x}} h_{m} x\right)}{A_{0} \sqrt{N_{j}}}\right) \\
& \times U\left(y-h_{m} x\right) .
\end{aligned}
$$

Considering the case of an idle jammer, the additive noise follows the normal distribution with $\sqrt{E_{x}} h_{m} x$ mean and $N_{0}$ variance. Hence, for all possible states of the jammer, we can write the comprehensive PDF of the received signal $y$ as

$$
\begin{aligned}
f_{Y}\left(y \mid \sqrt{E_{x}}, h_{m}, x\right)=\rho & \frac{\zeta_{j}^{2}}{\sqrt{N_{j}} A_{0}^{\zeta_{j}^{2}}}\left(\frac{y-\sqrt{E_{x}} h_{m} x}{\sqrt{N_{j}}}\right)^{\zeta_{j}^{2}-1} \Gamma\left(1-\zeta_{j}^{2}, \frac{\left(y-\sqrt{E_{x}} h_{m} x\right)}{A_{0} \sqrt{N_{j}}}\right) U\left(y-h_{m} x\right) \\
& +(1-\rho) \frac{1}{\sqrt{2 \pi N_{0}}} \exp \left(\frac{-\left(y-\sqrt{E_{x}} h_{m} x\right)^{2}}{2 N_{0}}\right) .
\end{aligned}
$$

This equation can be simplified for the possible values of $x$ as

$$
f_{Y}(y)=\left\{\begin{array}{lr}
\rho \frac{\zeta_{j}^{2}}{\sqrt{N_{j}} A_{0}^{\zeta_{j}^{2}}}\left(\frac{y}{\sqrt{N_{j}}}\right)^{\zeta_{j}^{2}-1} \Gamma\left(1-\zeta_{j}^{2}, \frac{y}{A_{0} \sqrt{N_{j}}}\right) U(y) \\
+(1-\rho) \frac{1}{\sqrt{2 \pi N_{0}}} \exp \left(\frac{-y^{2}}{2 N_{0}}\right), & \text { for } x=0, \\
\rho \frac{\zeta_{j}^{2}}{\sqrt{N_{j}} A_{0}^{\zeta_{j}^{2}}}\left(\frac{y-h_{m}}{\sqrt{N_{j}}}\right)^{\zeta_{j}^{2}-1} \Gamma\left(1-\zeta_{j}^{2}, \frac{y-h_{m}}{A_{0} \sqrt{N_{j}}}\right) U\left(y-h_{m}\right) \\
+(1-\rho) \frac{1}{\sqrt{2 \pi N_{0}}} \exp \left(\frac{-\left(y-h_{m}\right)^{2}}{2 N_{0}}\right), & \text { for } x=1 .
\end{array}\right.
$$


Furthermore, to determine the authentic information at the receiver, we utilize a threshold-based detection technique defined as

$$
y \stackrel{x=1}{\gtrless} y_{\text {th }} \text {. }
$$

Here, $y_{\text {th }}$ is the threshold which can be obtained by performing the Likelihood Ratio Test [36] on (17):

$$
y_{t h}=\sqrt{E_{x}}\left(\frac{h_{m}}{2}\right)-\frac{\rho}{1-\rho} \frac{N_{0}}{N_{j}} \sqrt{2 \pi N_{0}} .
$$

As discussed earlier, when the jammer is active, the jamming noise is far greater than the Gaussian noise, i.e., $N_{j} \gg N_{0}$; hence, the second term in (19) becomes negligible and can be neglected in further calculations. Thus, the effective threshold value for the received signal becomes

$$
y_{t h}=\sqrt{E_{x}}\left(\frac{h_{m}}{2}\right) .
$$

Now, considering both the transmitted signals to be equi-probable, we can determine the BER of the system in the presence of an active jammer from (9) as follows:

$$
B E R_{\mathrm{J}_{\text {active }}}\left(h_{m}\right)=\rho\left[0.5 \int_{y_{\text {th }}}^{\infty} f_{Y}(y \mid x=0) d x+0.5 \int_{0}^{y_{\text {th }}} f_{Y}(y \mid x=1) d x\right] .
$$

Taking into account the distribution of additive jamming noise and the presence of information signals, we can derive the BER contributed by the jammer using (17) in (21) as follows:

$$
B E R_{\mathrm{Jactive}}\left(h_{m}\right)=0.5 \rho \zeta_{j}^{2}\left(\frac{h_{m} \sqrt{\rho \gamma}}{2 A_{0}}\right)^{\zeta_{j}^{2}} \Gamma\left(-\zeta_{j}^{2} \frac{h_{m} \sqrt{\rho \gamma}}{2 A_{0}}\right) .
$$

The final expression of BER imparted by the jammer and Gaussian noise for the given FSO system can be obtained from (10) and (22):

$$
\operatorname{BER}\left(h_{m}\right)=0.5 \rho \zeta_{j}^{2}\left(\frac{h_{m} \sqrt{\rho \gamma}}{2 A_{0}}\right)^{\zeta_{j}^{2}} \Gamma\left(-\zeta_{j}^{2}, \frac{h_{m} \sqrt{\rho \gamma}}{2 A_{0}}\right)+(1-\rho) Q\left(\frac{h_{m} \sqrt{\kappa \gamma}}{2}\right) .
$$
RV, i.e.,

Now, the ABER can be calculated from (23) by integrating it over the PDF of the related

$$
\begin{aligned}
A B E R= & \int_{-\infty}^{+\infty}\left(0.5 \rho \zeta_{j}^{2}\left(\frac{h_{m} \sqrt{\rho \gamma}}{2 A_{0}}\right)^{\zeta_{j}^{2}} \Gamma\left(-\zeta_{j}^{2}, \frac{h_{m} \sqrt{\rho \gamma}}{2 A_{0}}\right)+(1-\rho) Q\left(\frac{h_{m} \sqrt{\kappa \gamma}}{2}\right)\right) \\
& \times f_{\left(h_{m}\right)}\left(h_{m}\right) d h_{m} \\
= & A B E R_{\mathrm{J}_{\text {active }}}+A B E R_{\mathrm{J}_{\text {idle }}}
\end{aligned}
$$

where

$$
A B E R_{\mathrm{Jactive}}=\int_{-\infty}^{+\infty} 0.5 \rho \zeta_{j}^{2}\left(\frac{h_{m} \sqrt{\rho \gamma}}{2 A_{0}}\right)^{\zeta_{j}^{2}} \Gamma\left(-\zeta_{j}^{2}, \frac{h_{m} \sqrt{\rho \gamma}}{2 A_{0}}\right) f_{\left(h_{m}\right)}\left(h_{m}\right) d h_{m}
$$

is the ABER contributed by the jammer, and

$$
A B E R_{\mathrm{J}_{\mathrm{idle}}}=\int_{-\infty}^{+\infty}(1-\rho) Q\left(\frac{h_{m} \sqrt{\kappa \gamma}}{2}\right) f_{\left(h_{m}\right)}\left(h_{m}\right) d h_{m},
$$

represents the ABER imparted by the Gaussian noise. To solve (25) and (26), we need the PDF for $h_{m}$ which is the maximum of $N$ fading coefficients for the channel suffering from saturated AT and PE conditions altogether. 


\subsection{PDF of Maximum of $N$ Channel Fading Coefficients}

We employ the order statistics [35] for multi-variables, defined for $N$ ordered i.i.d. RVs as

$$
h_{1} \leq \cdots \leq h_{k} \leq \cdots \leq h_{N}
$$

the density $f_{k}(h)$ of the $k$ th statistic is given by

$$
f_{k}(h)=\frac{N !}{(k-1) !(N-k) !} F_{h}^{k-1}(h)\left[1-F_{h}(h)\right]^{N-k} f_{h}(h),
$$

where $F_{h}(h)$ is the cumulative distribution function $(\mathrm{CDF})$, and $f_{h}(h)$ is the PDF of i.i.d. RVs. As we require the maximum of such $N$ RVs, we can substitute $k=N$ in (27), and thus we obtain

$$
f_{N}(h)=N F_{h}^{N-1}(h) f_{h}(h) .
$$

Note that the FSO links of the legitimate transmitter are i.i.d.; therefore, they will follow the same PDF, i.e., $f_{h_{u}}=f_{h_{u_{i}}}\left(h_{u_{i}}\right)$, and the same CDF, i.e., $F_{h_{u}}=F_{h_{u_{i}}}\left(h_{u_{i}}\right)$. Now, since we need the PDF of the maximum of $N$ fading coefficients experienced by the authentic MISO-FSO transmitter, we can replace $f_{h}(h)$ and $F_{h}(h)$ by $f_{h_{u}}\left(h_{u}\right)$ and $F_{h_{u}}\left(h_{u}\right)$, respectively, in (28). Thus, the CDF of $R V h_{u}$ can be obtained from its PDF, defined in (8) via

$$
F_{h_{u}}=\int_{-\infty}^{h_{u}} f_{h_{u}}\left(h_{u}\right) d h_{u}
$$

Solving (29) using (8), we obtain

$$
F_{h_{u}}=1-\exp \left(\frac{-h_{u}}{A_{0}}\right)+\frac{h_{u}}{A_{0}} E_{\zeta_{u}^{2}}\left(\frac{h_{u}}{A_{0}}\right),
$$

where $E_{n}(\cdot)$ is the generalized exponential integral of order $n$ [32]. Henceforth, the PDF of the best transmit link with saturated AT and PE conditions is calculated by using (30) and (8) in (28) as

$$
f_{h_{m}}\left(h_{m}\right)=N\left[1-\exp \left(\frac{-h_{m}}{A_{0}}\right)+\frac{h_{m}}{A_{0}} E_{\zeta_{u}^{2}}\left(\frac{h_{m}}{A_{0}}\right)\right]^{N-1} \frac{\zeta_{u}^{2}}{A_{0}^{\zeta_{u}^{2}}} h_{m}^{\zeta_{u}^{2}-1} \Gamma\left(1-\zeta_{u}^{2}, \frac{h_{m}}{A_{0}}\right),
$$

where $f_{h_{m}}\left(h_{m}\right)$ indicates the PDF of the best fading coefficient, i.e., $h_{m}$, out of $N$ available FSO links at the legitimate user. We hereby apply trinomial expansion [37] to convert the above expression as

$$
\begin{aligned}
f_{h_{m}}\left(h_{m}\right)= & N \sum_{p=0}^{N-1} \sum_{q=0}^{N-1-p} \frac{(N-1) !}{p ! q !(N-1-p-q) !} 1^{p}\left(-\exp \left(-\frac{h_{m}}{A_{0}}\right)\right)^{q} \\
& \times\left(\frac{h_{m}}{A_{0}} E_{\zeta_{u}^{2}}\left(\frac{h_{m}}{A_{0}}\right)\right)^{N-1-p-q} \frac{\zeta_{u}^{2}}{A_{0}^{\zeta_{u}^{2}}} h_{m}^{\zeta_{u}^{2}-1} \Gamma\left(1-\zeta_{u}^{2}, \frac{h_{m}}{A_{0}}\right) .
\end{aligned}
$$

Using property [34],

$$
E_{v}(z)=z^{v-1} \Gamma(1-v, z),
$$

in (32), we can write

$$
\begin{aligned}
f_{h_{m}}\left(h_{m}\right)= & N \sum_{p=0}^{N-1} \sum_{q=0}^{N-1-p} \frac{(N-1) !}{p ! q !(N-1-p-q) !} 1^{p}\left(-\exp \left(-\frac{h_{m}}{A_{0}}\right)\right)^{q}\left(\frac{h_{m}}{A_{0}}\right)^{N-1-p-q} \\
& \times\left(\left(\frac{h_{m}}{A_{0}}\right)^{\zeta_{u}^{2}-1} \Gamma\left(1-\zeta_{u}^{2}, \frac{h_{m}}{A_{0}}\right)\right)^{N-1-p-q} \frac{\zeta_{u}^{2}}{A_{0}^{\zeta_{u}^{2}}} h_{m}^{\zeta_{u}^{2}-1} \Gamma\left(1-\zeta_{u}^{2}, \frac{h_{m}}{A_{0}}\right)
\end{aligned}
$$


This is the final PDF for the maximum of $N$ RVs following the statistics defined in (8). Figure 3 shows the plots of PDF, obtained in (34), for different values of $N$. The matching of the simulation with analytical results proves the efficacy of the derived PDF.

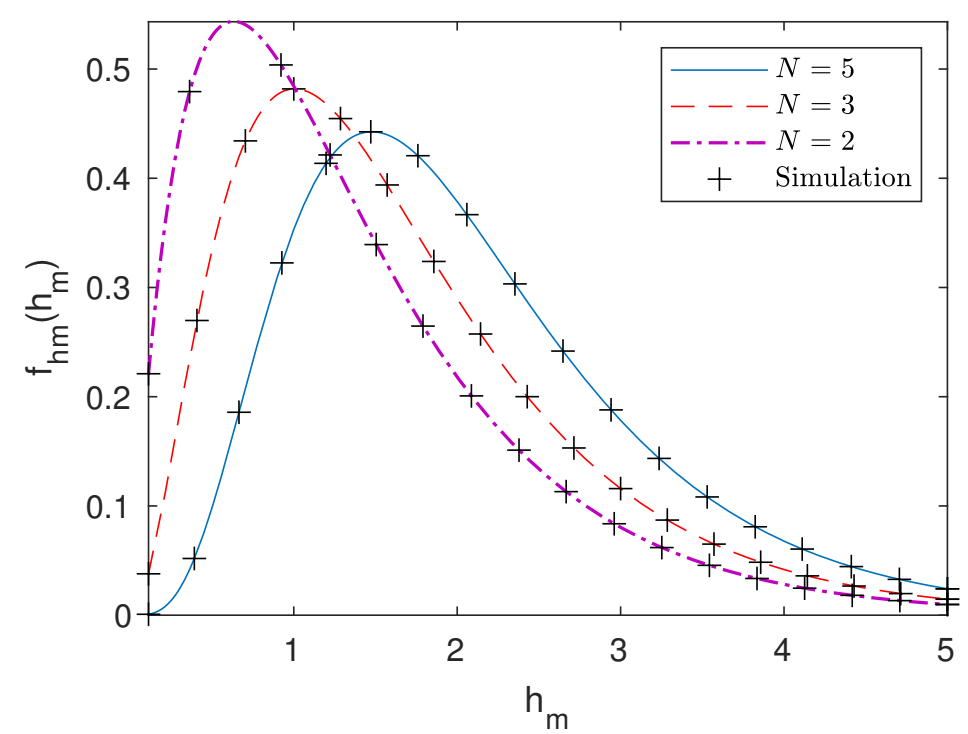

Figure 3. Plots of PDF of $h_{m}$ with different numbers of transmit apertures with $\zeta_{u}=3.4392$.

\subsection{ABER Calculation}

Now, we can calculate $A B E R_{\mathrm{J}_{\text {active }}}$ from (25) and (34) as

$$
\begin{aligned}
A B E R_{\mathrm{Jactive}}= & \int_{0}^{+\infty} 0.5 \rho \zeta_{j}^{2}\left(\frac{h_{m} \sqrt{\rho \gamma}}{2 A_{0}}\right)^{\zeta_{j}^{2}} \Gamma\left(-\zeta_{j}^{2}, \frac{h_{m} \sqrt{\rho \gamma}}{2 A_{0}}\right) N \sum_{p=0}^{N-1} \sum_{q=0}^{N-1-p} \frac{(N-1) !}{p ! q !(N-1-p-q) !} \\
& \times\left(-\exp \left(-\frac{h_{m}}{A_{0}}\right)\right)^{q}\left(\frac{h_{m}}{A_{0}}\right)^{N-1-p-q}\left(\left(\frac{h_{m}}{A_{0}}\right)^{\zeta_{u}^{2}-1} \Gamma\left(1-\zeta_{u}^{2}, \frac{h_{m}}{A_{0}}\right)\right)^{N-1-p-q} \\
& \times \frac{\zeta_{u}^{2}}{A_{0}^{\zeta_{u}^{2}}} \zeta_{m}^{\zeta_{u}^{2}-1} \Gamma\left(1-\zeta_{u}^{2}, \frac{h_{m}}{A_{0}}\right) d h_{m} .
\end{aligned}
$$

After rigorous algebra and using the property Equation (8.7.3) in [38],

$$
\Gamma(a, z)=\Gamma(a)-\sum_{l=0}^{\infty} \frac{(-1)^{l}}{l !} \frac{z^{a+l}}{(a+l)},
$$

for $l=0$, an upper bounded asymptotic expression of $A B E R_{\mathrm{J}_{\mathrm{active}}}$ can be obtained as

$$
\begin{aligned}
& A B E R_{\mathrm{J}_{\mathrm{active}}}=\int_{0}^{+\infty} 0.5 \rho \zeta_{u}^{2} \zeta_{j}^{2}\left(\frac{\sqrt{\rho \gamma}}{2}\right) \zeta_{p=0}^{\zeta_{j}^{2}} \sum_{q=0}^{N-1} \sum^{N-1-p}(-1)^{q} \frac{(N) !}{p ! q !(N-1-p-q) !} \\
& \times\left(\frac{\max \left(\Gamma\left(1-\zeta_{u}^{2}\right), 1\right)}{\max \left(\zeta_{u}^{2}-1,1\right)}\right)^{(N-p-q)}\left(\frac{h_{m}}{A_{0}}\right)^{\psi+\zeta_{j}^{2}-1} \exp \left(\frac{-h_{m} q}{A_{0}}\right) \Gamma\left(-\zeta_{j}^{2}, \frac{h_{m} \sqrt{\rho \gamma}}{2 A_{0}}\right) d h_{m}
\end{aligned}
$$

where $\delta=\min \left(1-\zeta_{u}^{2}, 1\right)$ and $\psi=(N-p-q)\left(\delta+\zeta_{u}^{2}\right)$. Employing the relation Equation (8.14.6) [38], given by

$$
\int_{0}^{+\infty} x^{a-1} \exp (-s x) \Gamma(b, x)=\frac{\Gamma(a+b)}{a(1+s)^{a+b}}{ }_{2} F_{1}\left(1, a+b ; 1+a ; \frac{s}{s+1}\right),
$$


where ${ }_{2} F_{1}(., \ldots, \ldots)$ represents the Gauss hypergeometric function [32], we obtain the final expression for the ABER contributed by the jammer:

$$
\begin{aligned}
A B E R_{\mathrm{Jactive}}= & 0.5 \rho \zeta_{u}^{2} \zeta_{j}^{2} \sum_{p=0}^{N-1} \sum_{q=0}^{N-1-p}(-1)^{q} \frac{(N) !}{p ! q !(N-1-p-q) !}\left(\frac{\max \left(\Gamma\left(1-\zeta_{u}^{2}\right), 1\right)}{\max \left(\zeta_{u}^{2}-1,1\right)}\right)^{(N-p-q)} \\
& \times \frac{\Gamma(\psi)}{\left(\zeta_{j}^{2}+\psi\right)\left(1+\frac{2 q}{\sqrt{\rho \gamma}}\right)^{\psi}}\left(\frac{2}{\sqrt{\rho \gamma}}\right)^{\psi}{ }_{2} F_{1}\left(1, \psi ; 1+\zeta_{j}^{2}+\psi ; \frac{2 q}{2 q+\sqrt{\rho \gamma}}\right) .
\end{aligned}
$$

Now, to compute the ABER due to Gaussian noise while the jammer is in an idle state, we use (34) in (26) and perform some algebra to obtain

$$
\begin{aligned}
A B E R_{\mathrm{J}_{\mathrm{idle}}}= & \int_{0}^{+\infty}(1-\rho) \frac{\zeta_{u}^{2}}{A_{0}} Q\left(\frac{h_{m} \sqrt{\kappa \gamma}}{2}\right) \sum_{p=0}^{N-1} \sum_{q=0}^{N-1-p} \frac{(N) !}{p ! q !(N-1-p-q) !}(-1)^{q} \\
& \times \exp \left(-\frac{h_{m} q}{A_{0}}\right)\left(\frac{h_{m}}{A_{0}}\right)^{\zeta_{u}^{2}(N-p-q)-1}\left(\Gamma\left(1-\zeta_{u}^{2}, \frac{h_{m}}{A_{0}}\right)\right)^{N-p-q} d h_{m} .
\end{aligned}
$$

Using (36) for $l=0$ in the above expression, we obtain an upper bounded asymptotic expression of $A B E R_{\mathrm{J}_{\text {idle }}}$ as

$$
\begin{aligned}
A B E R_{\mathrm{Jidle}}= & (1-\rho) \frac{\zeta_{u}^{2}}{A_{0}} \sum_{p=0}^{N-1} \sum_{q=0}^{N-1-p} \frac{(N) !}{p ! q !(N-1-p-q) !}(-1)^{q}\left(\frac{\max \left(\Gamma\left(1-\zeta_{u}^{2}\right), 1\right)}{\max \left(\zeta_{u}^{2}-1,1\right)}\right)^{(N-p-q)} \\
& \times \int_{0}^{+\infty} Q\left(\frac{h_{m} \sqrt{\kappa \gamma}}{2}\right) \exp \left(-\frac{h_{m} q}{A_{0}}\right)\left(\frac{h_{m}}{A_{0}}\right)^{\psi-1} d h_{m}
\end{aligned}
$$

Replacing $Q($.$) in the above expression via the following relation:$

$$
Q(a)=\frac{1}{2} \operatorname{erfc}\left(\frac{a}{\sqrt{2}}\right),
$$

where erfc(.) is the complementary error function [34], and employing [39], we can solve the integral in (41):

$$
\begin{aligned}
& A B E R_{\mathrm{J}_{\mathrm{idle}}}=0.5(1-\rho) \zeta_{u}^{2} \sum_{p=0}^{N-1} \sum_{q=0}^{N-1-p} \frac{(N) !}{p ! q !(N-1-p-q) !}(-1)^{q}\left(\frac{\max \left(\Gamma\left(1-\zeta_{u}^{2}\right), 1\right)}{\max \left(\zeta_{u}^{2}-1,1\right)}\right)^{(N-p-q)} \\
& \times\left(\frac{2 \sqrt{2}}{A_{0} \sqrt{\kappa \gamma}}\right)^{\psi} \frac{1}{\sqrt{\pi}}\left[\frac{1}{\psi} \Gamma\left(\frac{\psi+1}{2}\right){ }_{2} F_{2}\left(\frac{\psi+1}{2}, \frac{\psi}{2} ; \frac{1}{2}, \frac{\psi}{2}+1 ;\left(\frac{\sqrt{2} q}{A_{0} \sqrt{\kappa \gamma}}\right)^{2}\right)\right. \\
& \left.-\frac{1}{(\psi+1)}\left(\frac{2 \sqrt{2} q}{A_{0} \sqrt{\kappa \gamma}}\right) \Gamma\left(\frac{\psi}{2}+1\right){ }_{2} F_{2}\left(\frac{\psi+1}{2}, \frac{\psi}{2}+1 ; \frac{3}{2}, \frac{\psi+3}{2}+1 ;\left(\frac{\sqrt{2} q}{A_{0} \sqrt{\kappa \gamma}}\right)^{2}\right)\right]
\end{aligned}
$$

where ${ }_{2} F_{2}(., \ldots ; ., ;$.$) is the generalized hypergeometric function [32]. This culminates in the$ final expression of ABER contributed by Gaussian noise in the defined FSO system. The overall ABER of the defined MISO-FSO system can be written from (39) and (43) as

$$
A B E R=A B E R_{\mathrm{J}_{\text {active }}}+A B E R_{\mathrm{J}_{\mathrm{idle}}} .
$$

Remark 1. This is an exhaustive expression of ABER for an $N \times 1$ MISO-FSO system employing transmit aperture selection, suffering from a jammer and Gaussian noise, while both the legitimate transmitter and the jammer experience channel fading due to saturated AT and PE conditions.

\subsection{Coding Gain and Diversity Order}

To determine the coding gain and diversity order of the defined FSO system, we need to evaluate the ABER expression (44) at a very high value of $\gamma$. At such values of $\gamma$, 
the dominating terms are affiliated to the lowest exponent of $\gamma$. We take two active and idle jammer cases, respectively, to understand the difference between the jamming and Gaussian noise.

Case I: For an active jammer scenario, we put $\gamma \rightarrow \infty$ in (39) and collect the dominating terms as

$$
\lim _{\gamma \rightarrow \infty} A B E R_{\mathrm{J}_{\mathrm{active}}}=0.5 \rho \zeta_{u}^{2} \zeta_{j}^{2} N\left(\frac{\max \left(\Gamma\left(1-\zeta_{u}^{2}\right), 1\right)}{\max \left(\zeta_{u}^{2}-1,1\right)}\right)^{N} \frac{\Gamma\left(N\left(\delta+\zeta_{u}^{2}\right)\right)}{\left(\zeta_{j}^{2}+N\left(\delta+\zeta_{u}^{2}\right)\right)}\left(\frac{2}{\sqrt{\rho \gamma}}\right)^{N\left(\delta+\zeta_{u}^{2}\right)} .
$$

Comparing (45) with (12), we obtain the coding gain as

$$
G_{c}=\left[0.5 \rho \zeta_{u}^{2} \zeta_{j}^{2} N\left(\frac{\max \left(\Gamma\left(1-\zeta_{u}^{2}\right), 1\right)}{\max \left(\zeta_{u}^{2}-1,1\right)}\right)^{N} \frac{\Gamma\left(N\left(\delta+\zeta_{u}^{2}\right)\right)}{\left(\zeta_{j}^{2}+N\left(\delta+\zeta_{u}^{2}\right)\right)}\left(\frac{2}{\sqrt{\rho}}\right)^{N\left(\delta+\zeta_{u}^{2}\right)}\right]^{\frac{N\left(\delta+\zeta_{u}^{2}\right)}{2}}
$$

and the diversity order $G_{d}$ as $0.5 N\left(\delta+\zeta_{u}^{2}\right)$. Thus, the maximum diversity order that the system can attain is $0.5 \mathrm{~N}$.

Remark 2. The transmit aperture selection allows the FSO system corrupted by a jammer to attain the same diversity order as an MISO-FSO system [12], while considerably saving the power requirements of $N-1$ transmitters. terms as

Case II: For an idle jammer scenario, we put $\gamma \rightarrow \infty$ in (43) and obtain the dominating

$$
\begin{aligned}
\lim _{\gamma \rightarrow \infty} A B E R_{\mathrm{J}_{\mathrm{idle}}}= & 0.5(1-\rho) \zeta_{u}^{2} N\left(\frac{\max \left(\Gamma\left(1-\zeta_{u}^{2}\right), 1\right)}{\max \left(\zeta_{u}^{2}-1,1\right)}\right)^{N}\left(\frac{2 \sqrt{2}}{A_{0} \sqrt{\kappa \gamma}}\right)^{N\left(\delta+\zeta_{u}^{2}\right)} \frac{1}{\sqrt{\pi} N\left(\delta+\zeta_{u}^{2}\right)} \\
& \times \Gamma\left(\frac{N\left(\delta+\zeta_{u}^{2}\right)+1}{2}\right) .
\end{aligned}
$$

Again, comparing (47) with (12), we obtain the coding gain as

$$
\begin{aligned}
G_{c}= & {\left[0.5(1-\rho) \zeta_{u}^{2} N\left(\frac{\max \left(\Gamma\left(1-\zeta_{u}^{2}\right), 1\right)}{\max \left(\zeta_{u}^{2}-1,1\right)}\right)^{N}\left(\frac{2 \sqrt{2}}{A_{0} \kappa}\right)^{N\left(\delta+\zeta_{u}^{2}\right)} \frac{1}{\sqrt{\pi} N\left(\delta+\zeta_{u}^{2}\right)}\right.} \\
& \left.\times \Gamma\left(\frac{N\left(\delta+\zeta_{u}^{2}\right)+1}{2}\right)\right]^{0.5 N\left(\delta+\zeta_{u}^{2}\right)},
\end{aligned}
$$

and the diversity order is $0.5 N\left(\delta+\zeta_{u}^{2}\right)$, which is the maximum diversity that can be achieved for any $N \times 1$ MISO system.

\section{Numerical Results and Discussions}

This section illustrates the impact of aperture selection on the error performance of an MISO-FSO system, transmitting OOK signals from $N$ user transmitters, and under attack from a jammer; the legitimate signals and jamming signal suffer from channel fading following negative exponential statistics with PE. The derived expressions in the preceding sections are utilized to arrive at the analytical results; simulation results are realized by Monte-Carlo simulation after modeling the defined channel fading conditions. At the receiver, we take $\kappa$ to be 10 in all the plotted graphs. The PE parameter is taken to be 4.5856 and 1.7196 for user transmitters and jammers, respectively. The PE parameter of the user transmitter is higher to represent the condition that user transmitters are designed to be more aligned to the receiver. In all figures, the x-axis denotes the average SJNR per bit.

In Figure 4, we plot the analytical expression for ABER under the condition of an always-on jammer $(\rho=1)$ with a varying number of user transmitters deployed from (39). 
The results from the simulation are also plotted on the same graph. The match between the analytical and simulation plots proves the accuracy of the derived expression. It can be observed that as the number of user transmitters increases, the ABER performance of the system improves significantly. At $\gamma=20 \mathrm{~dB}$, we can see that the ABER drops drastically as $1.668 \times 10^{-2}, 3.587 \times 10^{-3}, 1.132 \times 10^{-3}, 2.91 \times 10^{-4}, 1.8 \times 10^{-4}$ for $N=2,3,5,7$, and 8 , respectively. For comparison purposes, we also plot the simulation result of a single-input single-output (SISO)-FSO system. It is noted that for $\gamma=30 \mathrm{~dB}$, transmit aperture selection reduces the bit error from $2.355 \times 10^{-2}$ to $1.664 \times 10^{-4}$, by allowing the system to choose the maximum of three-channel gains. Moreover, we observe that with an increase in $\gamma$, the improvement in bit error escalates. The diversity order of the plots for $N=2$ and 3 are 0.9970 and 1.494, respectively, and thus in agreement with the derived results.

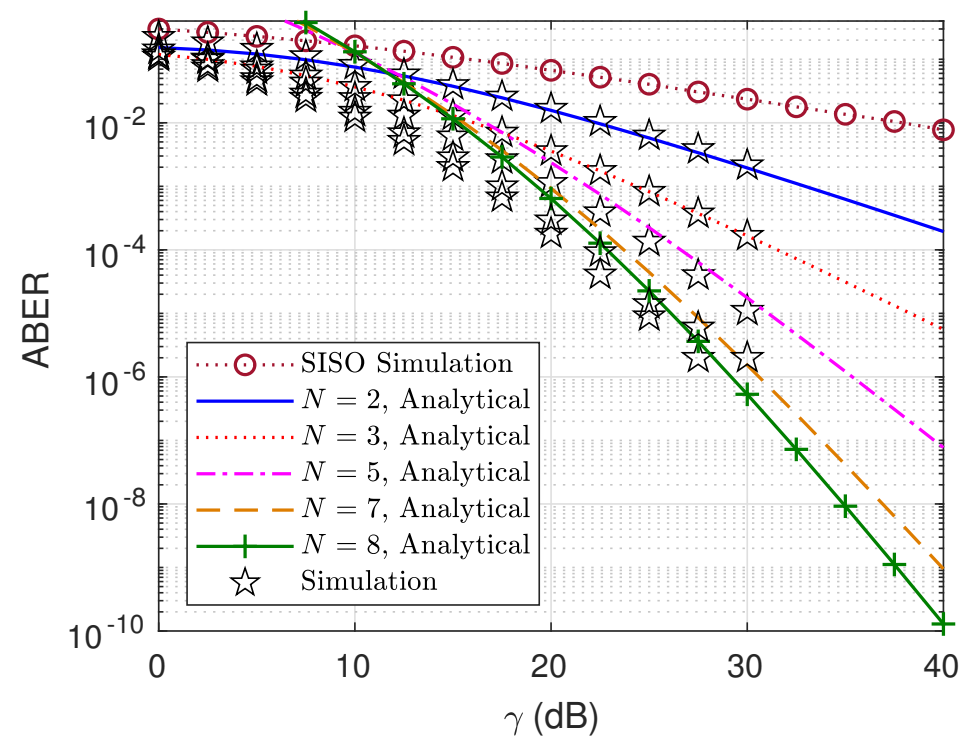

Figure 4. ABER versus SJNR (dB) curves for FSO system with varying number of user transmitters in presence of jammer $(\rho=1)$.

Remark 3. The aforementioned discussion confirms the efficiency of the transmit aperture selection technique to improve the error performance of a jammer-attacked FSO system, while also significantly reducing the system's power requirements to operate multiple transmitters.

Figure 5 plots the error performance of a $2 \times 1$ FSO system for different degrees of jammer activity; analytical results are obtained from (44). The figure verifies that as the jammer activity increases, the system's error performance degrades. When the value of $\rho$ widens from 0.05 to 1 at $\gamma=10 \mathrm{~dB}$, the bit error also jumps up from 0.02916 to 0.08141 . This demonstrates the grievous effect of a jammer as compared to the Gaussian noise. Moreover, as the $\rho$ increases from 0.1 to 0.3 , the ABER inflates from 0.03704 to 0.05567 , and an increase in $\rho$ from 0.3 to 1 results in an ABER hike from 0.05567 to 0.08141 ; this shows that as the jammer's activity increases, the effect of Gaussian noise on ABER diminishes. Furthermore, it can be noted that the difference in the error performance reduces as the $\gamma$ increases. This is evident from the gap of $9.108 \times 10^{-2}$ and $9.29 \times 10^{-4}$ at $\gamma=5 \mathrm{~dB}$ and $27.5 \mathrm{~dB}$ when the $\rho$ rises from 0.05 to 1 . This is because as the legitimate signals are transmitted with higher power, the effect of the varying degree of the jammer's activity reduces on the bit error performance. 


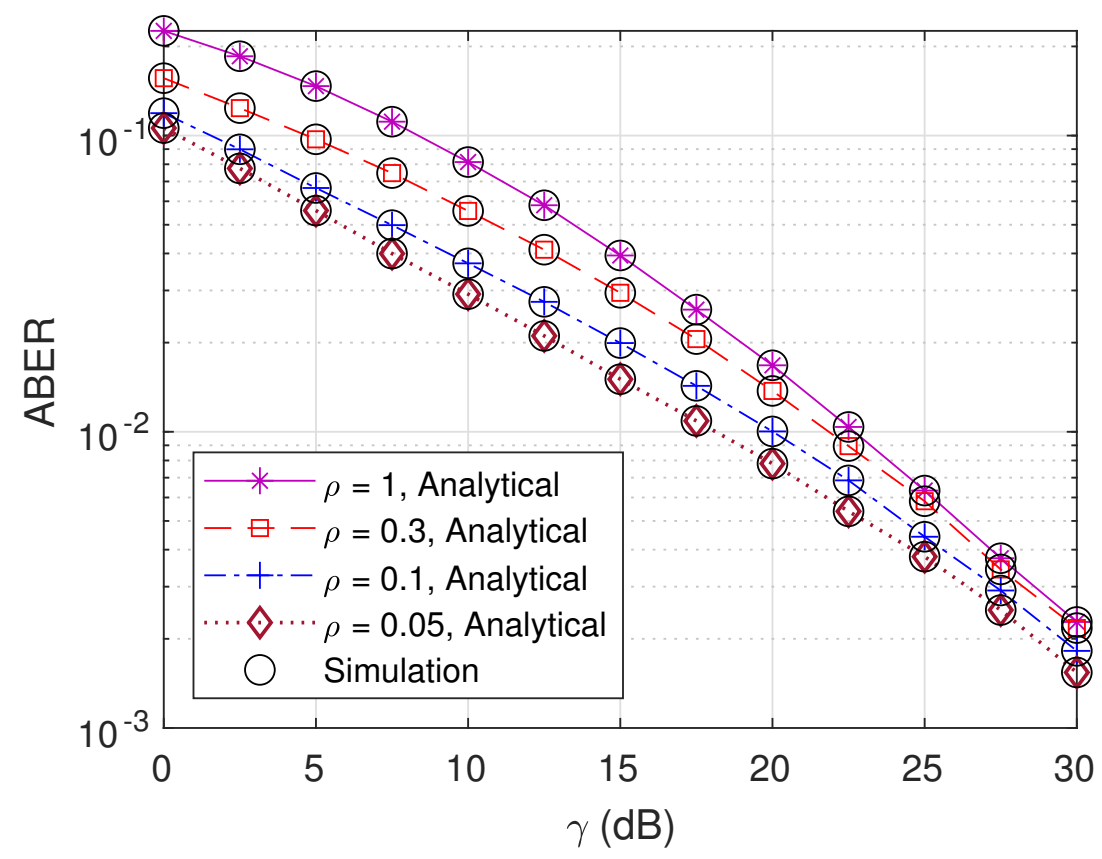

Figure 5. ABER versus SJNR (dB) curves for FSO system with $N=2$, with varying degree of jammer's activity.

Remark 4. The prior analysis shows that the jammer has a more pronounced effect on the ABER of the FSO system than the Gaussian noise. Additionally, at a considerably high value of $\gamma$, the error performance becomes insulated from any variation in $\rho$.

Figure 6 demonstrates the effect of an absent and active jammer. We can notice that an always-on jammer has a more adverse influence on the system's performance than Gaussian noise. At $\gamma=20 \mathrm{~dB}$, for $N=2$, the ABER increases from $5.627 \times 10^{-3}$ to $1.667 \times 10^{-2}$ as $\rho$ increases from 0 to 1 . Moreover as the $\gamma$ increases, the difference in the toll the two noises take on the performance widens. When two transmit apertures are employed, for $\rho=0$ and 1, the bit error rate has a difference of $2.094 \times 10^{-2}$ and $1.874 \times 10^{-3}$ at $\gamma=15 \mathrm{~dB}$ and $30 \mathrm{~dB}$, respectively. It is also observed that this divergence inflates as $N$ increases. At $\gamma=25 \mathrm{~dB}$, the gap between the bit error contributed by the jammer and the Gaussian noise is $4.931 \times 10^{-3}$ and $6.468 \times 10^{-4}$ for $N=2$ and 3, respectively. Hence, the transmit aperture selection technique performs substantially better in the absence of a jammer.

In Figure 7, we plot the ABER values of the FSO system with $N$ equal to 2 and 3, while varying the PE severity faced by the jammer. It can be observed from the figure that as the value of $\zeta_{j}$ increases, the PE suffered by the jammer reduces, and thus it disrupts the regular communication more strongly. It can be noticed from the figure that for ABER $=10^{-2}$, as the PE parameter changes from 0.5708 to $1.7196, \gamma$ has to be increased by $10 \mathrm{~dB}$ for the FSO system with two transmit apertures. However, under the same conditions, if the number of transmit apertures is increased to three, then only $7.5 \mathrm{~dB}$ of additional SJR is required to attain $\mathrm{ABER}=10^{-2}$. This also proves that $\mathrm{PE}$ conditions are more relenting when $N$ increases. 


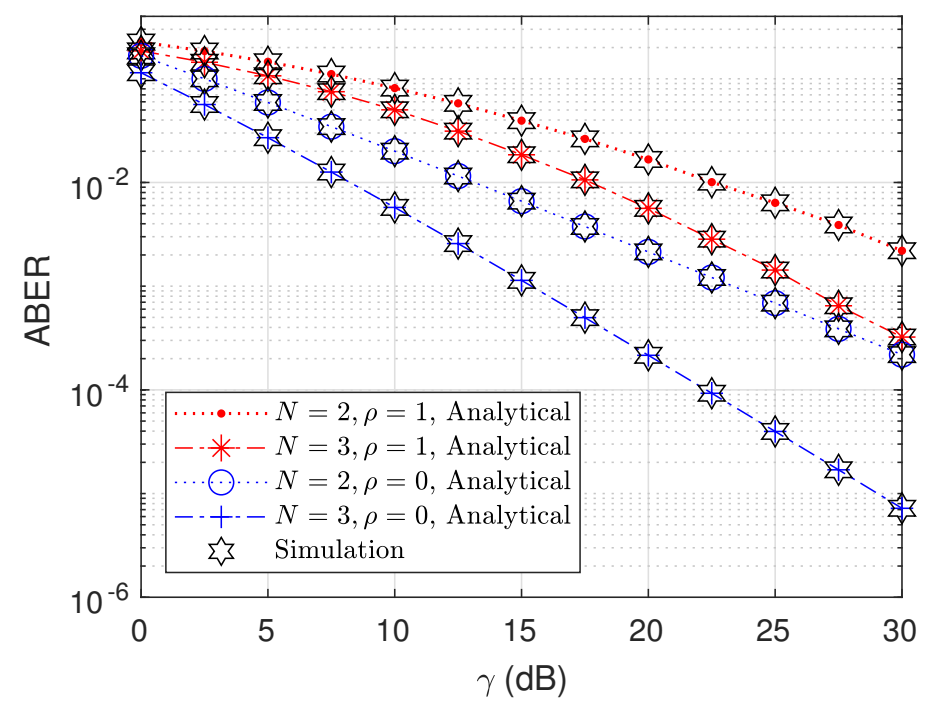

Figure 6. ABER versus SJNR $(\mathrm{dB})$ curves for the FSO systems having $N=2$ and 3 in the presence and absence of the jammer.

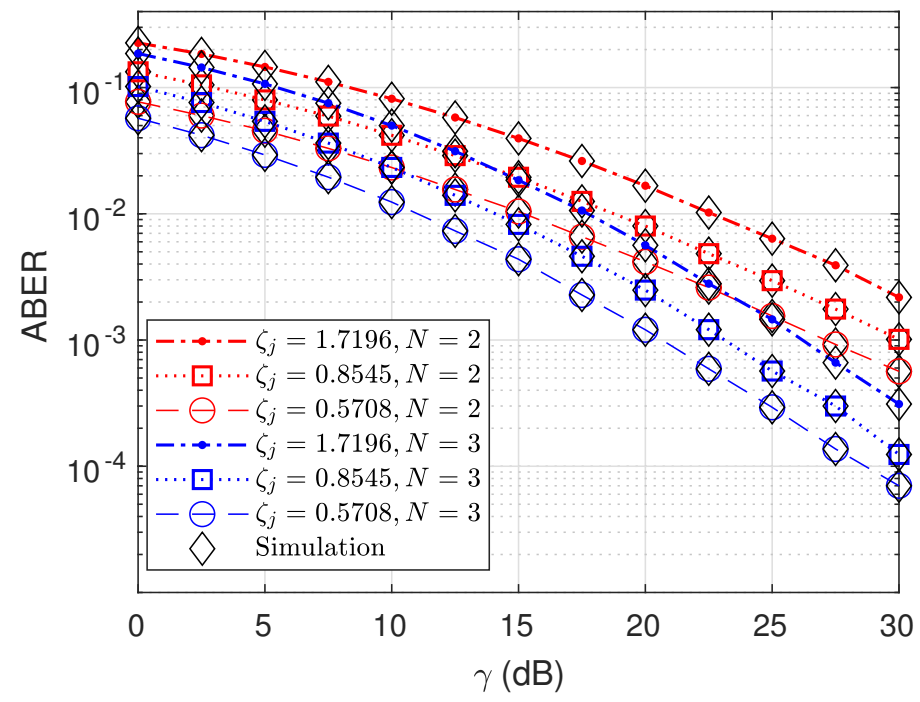

Figure 7. ABER versus SJNR (dB) curves for FSO systems having $N=2$ and 3 with varying PE severity in presence of jammer $(\rho=1)$.

Remark 5. The preceding discussion proves that serious PE conditions in the jamming link improve the ABER of the FSO system. Moreover, the bit error performance enhances as $N$ increases for an arbitrary value of $\zeta_{j}$.

In Figure 8, we present the comparison of different existing techniques with the transmit aperture selection technique in their resilience to combat the jamming noise. We plot the simulations of the OSSK scheme and RC scheme employed in a $2 \times 1$ FSO system under attack from a jammer. The channel is modeled for saturated AT and PE conditions for all the schemes. The PE parameters for an authorized transmitter and jammer are taken to be 1.5285 and 0.5708 . It is clear from the plot that the transmit aperture selection technique outperforms RC and OSSK significantly. At $\gamma=20 \mathrm{~dB}$, the error rates for OSSK, $\mathrm{RC}$, and aperture selection are $0.0312,0.0139$, and 0.0082 , respectively. The $\mathrm{RC}$ and aperture selection have a $3 \mathrm{~dB}$ gap between them, with the same diversity order of 1 , whereas OSSK has a diversity order of 0.5 , and hence the gap widens as $\gamma$ increases. 
Numerical Studies over Gamma-Gamma Fading: Now, we study the impact of aperture selection on an FSO system when the channel is modeled by GG statistics, augmented with PE. The calculations carried out in the previous section are extended for GG fading to scrutinize the defined system for various AT regimes. As it is a very grueling task to analyze an FSO system employing a transmit aperture selection technique to counter a jammer, we provide the simulation results in conformity with the numerical results obtained in the previous sections. The PDF of the GG channel with the combined effect of AT and PE is calculated in [3] as

$$
f_{h}(h)=\frac{\alpha \beta \xi^{2}}{A_{0} \Gamma(\alpha) \Gamma(\beta)} G_{1,3}^{3,0}\left(\frac{\alpha \beta h}{A_{0}} \mid \begin{array}{c}
\xi^{2}-1, \alpha-1, \beta-1
\end{array}\right),
$$

where $G_{p, q}^{m, n}(. \mid)$ is the Meijer-G function (Section 2.24 in [32]). The parameters $\alpha$ and $\beta$ characterize the irradiance fluctuations [2]. In this work, we consider moderate and strong AT regimes with $\alpha$ and $\beta$ equal to $\{4,1.9\}$ and $\{4.2,1.4\}$, respectively. Since BER signifies the system's performance, we present the numerical results of bit error of the system with varying AT and PE conditions for different degrees of the jammer's activity.

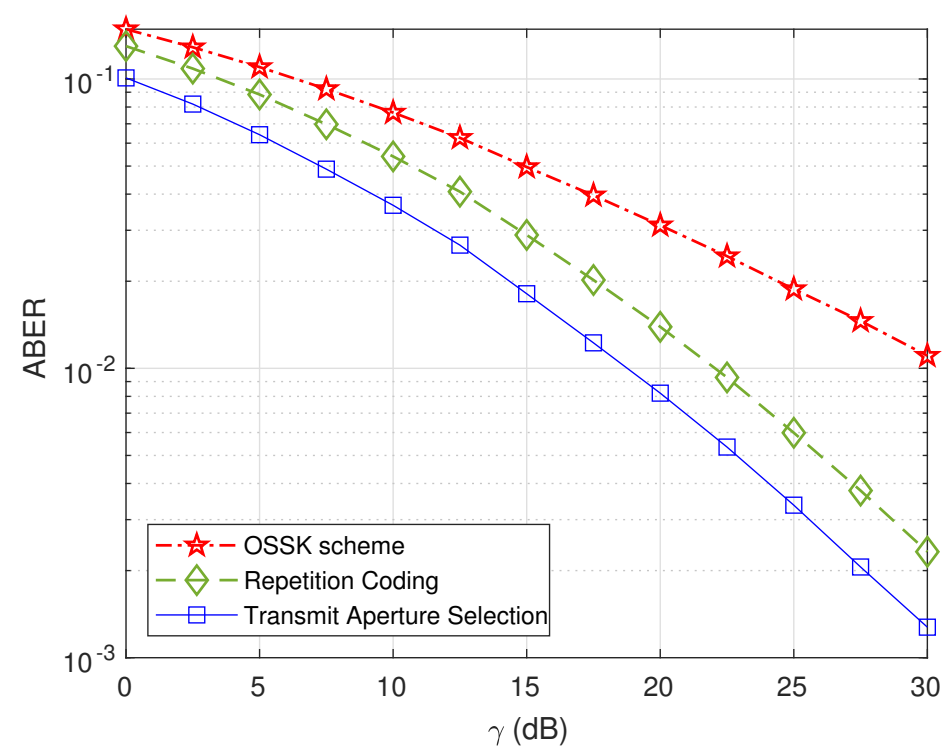

Figure 8. ABER versus SJNR (dB) curves for FSO system with $N=2, \zeta_{s}=1.5285, \zeta_{j}=0.5708$, and $\rho=1$.

Figure 9 presents the simulation results obtained after performing Monte-Carlo simulations modeling the GG fading channel following (49) with altering AT conditions and an active jammer. It demonstrates the improvement in bit error as $N$ increases for both strong and moderate AT regimes. For the moderate AT condition, ABER improves remarkably from $1 \times 10^{-2}$ to $3.76 \times 10^{-3}$ and $1.76 \times 10^{-3}$ for $N=2,3$, and 4 , respectively, for $\gamma=20 \mathrm{~dB}$. It can be seen from the figure that the advantages of transmit aperture selection are noticed for GG channel fading as well. As the SJNR increases, the effect becomes more striking. Moreover, as the AT conditions worsen, the system's performance takes a toll. 


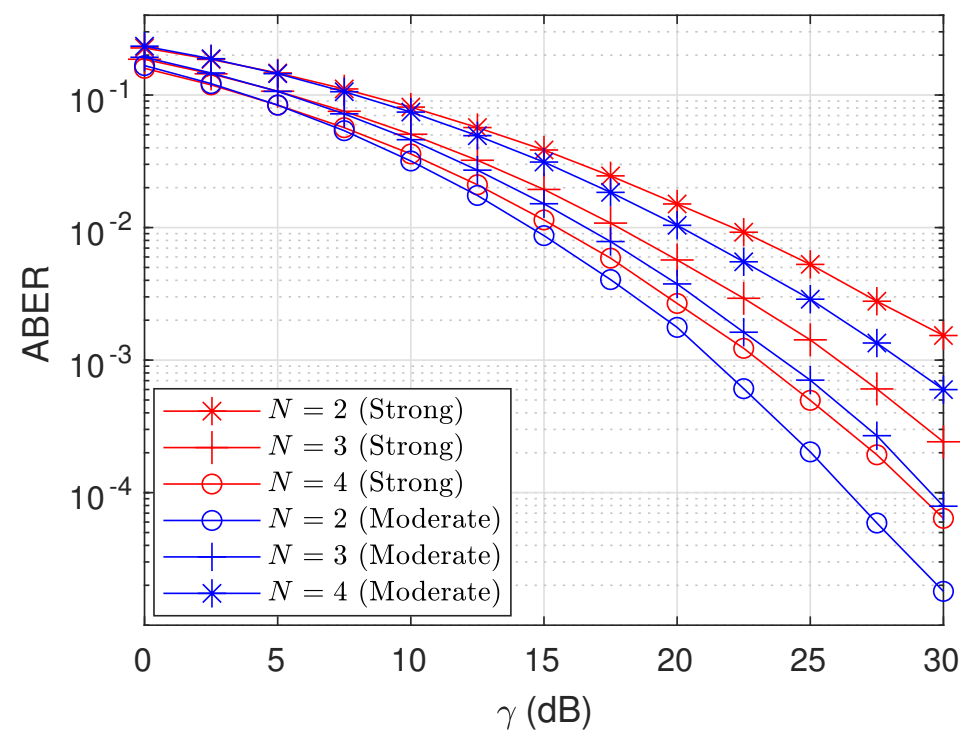

Figure 9. ABER versus SJNR (dB) curves for FSO systems having $N=2$ and 3 with varying AT conditions in presence of jammer $(\rho=1)$.

\section{Conclusions}

We have studied an approach to mitigate the jammer attack on an $N \times 1$ FSO system in this work via employing an aperture selection technique. The system suffers from jamming and Gaussian noise simultaneously, while the transmission channel models saturated AT accompanied with PE. An exhaustive expression of ABER encompassing both types of noise has been derived. The simulation results are plotted with analytical results to confirm the veracity of the derived expression. It has been discerned that as the number of user transmitters increases, the bit error performance of the system improves extensively. This model saves the power demand of allowing $N$ transmitters to operate en masse. Moreover, the system achieves the same diversity order as an MISO-FSO system with a jammer. We also notice that as the degree of the jammer's activity increases, the ABER reduces. However, raising the SJNR (dB) insulates the system from reacting to variation in $\rho$. Furthermore, variation in PE conditions in the jamming link is also examined. We observe that as the PE increases for the jammer, the system's performance improves. Moreover, expanding the number of user transmitters lowers the bit error of the system for any value of the PE parameter.

Author Contributions: Conceptualization, I.C. and M.R.B.; methodology, I.C. and M.R.B.; software, I.C.; validation, M.R.B.; formal analysis, I.C.; investigation, I.C. and M.R.B.; resources, M.R.B.; data curation, M.R.B.; writing—original draft preparation, I.C.; writing—review and editing, M.R.B.; visualization, M.R.B.; supervision, M.R.B.; funding acquisition, M.R.B. All authors have read and agreed to the published version of the manuscript.

Funding: This work was supported by the Brigadier Bhopinder Singh Chair, Indian Institute of Technology-Delhi, New Delhi, India.

Institutional Review Board Statement: Not applicable.

Informed Consent Statement: Not applicable.

Data Availability Statement: Data sharing is not applicable to this article.

Conflicts of Interest: The authors declare no conflict of interest. 


\section{References}

1. Andrews, L.C.; Phillips, R.L. Laser Beam Propagation through Random Media; SPIE: Bellingham, WA, USA, 2005.

2. Ghassemlooy, Z.; Popoola, W.; Rajbhandari, S. Optical Wireless Communications: System and Channel Modelling With MATLAB; CRC Press: Boca Raton, FL, USA, 2013.

3. Bhatnagar, M.R.; Ghassemlooy, Z. Performance analysis of gamma-gamma fading FSO MIMO links with pointing errors. IEEE/OSA J. Light. Technol. 2016, 34, 2158-2169. [CrossRef]

4. Bayaki, E.; Schober, R.; Mallik, R.K. Performance analysis of MIMO free-space optical systems in gamma-gamma fading. IEEE Trans. Commun. 2009, 57, 3415-3424. [CrossRef]

5. Letzepis, N.; Fabregas, A.G. Outage probability of the Gaussian MIMO free-space optical channel with PPM. IEEE Trans. Commun. 2009, 57, 3682-3690. [CrossRef]

6. Bhatnagar, M.R.; Anees, S. On the Performance of Alamouti Scheme in Gamma-Gamma Fading FSO Links With Pointing Errors. IEEE Wirel. Commun. Lett. 2015, 4, 94-97. [CrossRef]

7. Abaza, M.R.; Mesleh, R.; Mansour, A.; Aggoune, E.H.M. Diversity techniques for a free-space optical communication system in correlated log-normal channels. Opt. Eng. 2014, 53, 016102. [CrossRef]

8. Garcia-Zambrana, A.; Castillo-Vazquez, C.; Castillo-Vazquez, B.; Hiniesta-Gomez, A. Selection Transmit Diversity for FSO Links Over Strong Atmospheric Turbulence Channels. IEEE Photonics Technol. Lett. 2009, 21, 1017-1019. [CrossRef]

9. García-Zambrana, A.; Castillo-Vázquez, B.; Castillo-Vázquez, C. Asymptotic error-rate analysis of FSO links using transmit laser selection over Gamma-Gamma atmospheric turbulence channels with pointing errors. Opt. Exp. 2012, 20, 2096--2109. [CrossRef]

10. Samimi, H. Performance analysis of free-space optical links with transmit laser selection diversity over strong turbulence channels. IET Commun. 2011, 5, 1039-1043. [CrossRef]

11. Bhatnagar, M.R. A One Bit Feedback Based Beamforming Scheme for FSO MISO System Over Gamma-Gamma Fading. IEEE Trans. Commun. 2015, 63, 1306-1318. [CrossRef]

12. Paul, P.; Bhatnagar, M.R.; Jaiswal, A. Jamming in free space optical systems: mitigation and performance evaluation. IEEE Trans. Commun. 2020, 68, 1631-1647. [CrossRef]

13. Khalighi, M.A.; Uysal, M. Survey on Free Space Optical Communication: A Communication Theory Perspective. IEEE Commun Surv. Tut. 2014, 16, 2231-2258. [CrossRef]

14. Paul, P.; Bhatnagar, M.R.; Jaiswal, A. Alleviation of Jamming in Free Space Optical Communication over Gamma-Gamma Channel with Pointing Errors. IEEE Photonics J. 2019, 11, 1-18. [CrossRef]

15. MISSION TO EARTH: Optical Payload for Lasercomm Science. Available online: https://www.jpl.nasa.gov/missions/opticalpayload-for-lasercomm-science-opals/ (accessed on 10 July 2021).

16. European Data Relay Satellite System (EDRS). Available online: https://artes.esa.int/edrs-global (accessed on 10 July 2021).

17. SpaceBelt Data Security as a Service. Available online: http://spacebelt.com/ (accessed on 10 July 2021).

18. Connect People Everywhere. Available online: https:/ /loon.com/ (accessed on 10 July 2021).

19. Gajić, A.; Radovanović, J.; Vuković, N.; Milanović, V.; Boiko, D.L. Theoretical approach to quantum cascade micro-laser broadband multimode emission in strong magnetic fields. Phys. Lett. A 2021, 387, 127007. [CrossRef]

20. Garlinska, M.; Pregowska, A.; Masztalerz, K.; Osial, M. From Mirrors to Free-Space Optical Communication-Historical Aspects in Data Transmission. Future Internet 2020, 12, 179. [CrossRef]

21. Lionis, A.; Peppas, K.; Nistazakis, H.E.; Tsigopoulos, A.D.; Cohn, K. Experimental Performance Analysis of an Optical Communication Channel over Maritime Environment. Electronics 2020, 9, 1109. [CrossRef]

22. Wang, Y.; Xu, H.; Li, D.; Wang, R.; Jin, C.; Yin, X.; Gao, S.; Mu, Q.; Xuan, L.; Cao, Z. Performance analysis of an adaptive optics system for free-space optics communication through atmospheric turbulence. Sci. Rep. 2018, 8, 1124. [CrossRef]

23. Cheng, M.; Guo, L.; Zhang, Y.; Li, J. Selection combining optimization for FSO links over exponentiated Weibull fading channels. In Proceedings of the 2016 25th Wireless and Optical Communication Conference (WOCC), Chengdu, China, 21-23 May 2016; pp. 1-4. [CrossRef]

24. Moradi, H.; Refai, H.H.; LoPresti, P.G. Circular MIMO FSO Nodes With Transmit Selection and Receive Generalized Selection Diversity. IEEE Trans. Veh. Technol. 2012, 61, 1174-1181. [CrossRef]

25. Sharma, S.; Tan, J.; Madhukumar, A.; Swaminathan, R. Switching-Based Transmit Antenna/Aperture Selection in a MISO Hybrid FSO/RF System. In Proceedings of the 2018 IEEE Global Communications Conference (GLOBECOM), Abu Dhabi, United Arab Emirates, 9-13 December 2018; pp. 1-6. [CrossRef]

26. Zou, D.; Gong, C.; Xu, Z. Secrecy rate of MISO optical wireless scattering communications. IEEE Trans. Commun. 2018, 66, 225-238. [CrossRef]

27. Chauhan, I.; Paul, P.; Bhatnagar, M.R.; Nebhen, J. Performance of optical space shift keying under jamming. Appl. Opt. 2021, 60, 1856-1863. [CrossRef]

28. Yang, H.; Zhao, X.; Yao, Q.; Yu, A.; Zhang, J.; Ji, Y. Accurate Fault Location using Deep Neural Evolution Network in Cloud Data Center Interconnection. IEEE Trans. Cloud Comput. 2020. [CrossRef]

29. Yao, Q.; Yang, H.; Bao, B.; Yu, A.; Zhang, J.; Cheriet, M. Core and Spectrum Allocation Based on Association Rules Mining in Spectrally and Spatially Elastic Optical Networks. IEEE Trans. Commun. 2021, 69, 5299-5311. [CrossRef] 
30. Verma, G.D.; Mathur, A. Performance improvement of FSO communication systems using hybrid-ARQ protocols. Appl. Opt. 2021, 60, 5553-5563. [CrossRef] [PubMed]

31. Gradshteyn, I.S.; Ryzhik, I.M. Table of Integrals, Series, and Products, 7th ed.; Elsevier/Academic Press: Amsterdam, The Netherlands, 2007.

32. Prudnikov, A.P.; Brychkov, Y.A.; Marichev, O.I. Integrals and Series; Gordon and Breach: New York, NY, USA; London, UK, 1990; Volume 3.

33. Touati, A.; Abdaoui, A.; Touati, F.; Uysal, M.; Bouallegue, A. On the effects of combined atmospheric fading and misalignment on the hybrid FSO/RF transmission. IEEE/OSA J. Opt. Commun. Netw. 2016, 8, 715-725. [CrossRef]

34. Abramowitz, M.I.S. Handbook of Mathematical Functions with Formulas, Graphs, and Mathematical Tables; Dover: New York, NY, USA, 1964.

35. Papoulis, A.; Pillai, S.U. Probability, Random Variables and Stochastic Processes, 4th ed.; Tata McGraw-Hill: Boston, MA, USA, 2008.

36. Trees, H.L.V. Detection, Estimation, and Modulation Theory, Part I; John Wiley and Sons: New York, NY, USA, 2004.

37. Koshy, T. Discrete Mathematics with Applications; Academic Press: Amsterdam, The Netherlands; Boston, MA, USA, 2004.

38. Olver, F.W.J.; Lozier, D.W.; Boisvert, R.F.; Clark, C.W. NIST Handbook of Mathematical Functions; Cambridge University Press: New York, NY, USA, 2010.

39. Wolfram Research, Inc. Available online: http:/ / functions.wolfram.com/06.27.21.0133.01 (accessed on 15 August 2021). 